\title{
Circularity in relative pitch judgments for inharmonic complex tones: The Shepard demonstration revisited, again
}

\author{
EDWARD M. BURNS \\ Department of Audiology and Speech Sciences, Purdue University, West Lafayette, Indiana 47907
}

\begin{abstract}
The Shepard illusion, in which the presentation of a cyclically repetitive sequence of complex tones composed of partials separated by octave intervals (Shepard, 1964) gives the illusion of an endlessly increasing sequence of pitch steps, is often cited as evidence for octave equivalence. In this paper, evidence is presented which demonstrates that this illusion can be produced using (inharmonic) complex tones whose partials are separated by equal ratios other than octaves. Therefore, the illusion is not evidence for octave equivalence.
\end{abstract}

A number of authors (e.g., Deutsch, 1969; Idson \& Massaro, 1978; Krumhansl \& Shepard, 1979; Revez, 1954; Shepard, 1964) have proposed that pitch is best represented by a bidimensional model rather than by the unidimensional model derived from psychophysical scaling experiments (e.g., Stevens \& Volkmann, 1940). The two aspects of this bidimensional model are: "pitch height," which, for a pure tone, is a monotonic function of stimulus frequency (e.g., Stevens \& Volkmann, 1940); and tone "chroma," which defines tones whose frequencies stand in a frequency ratio of $2: 1$ as being in some sense equivalent (octave similarity). Bidimensional models are often graphically represented by a helix in which pitch height is indicated by the projection on the vertical axis (one turn of the helix per octave) and chroma by the projection on the circular plane (e.g., Revez, 1954). The bidimensional model is, of course, consistent with the musical scales of most cultures (Burns \& Ward, 1981), in which tones separated by an octave are considered in some respects musically equivalent and are given the same letter or mnemonic label.

Among experiments that have provided evidence for octave similarity or octave equivalence, those that have attempted to separate observers into "musically trained" and "musically untrained" categories have typically found a high correlation between these categories and the type of results obtained. Musically trained observers tend to show a high degree of octave equivalence, whereas musically untrained observers show little or none (Allen, 1967; Krumhansl \& Shepard, 1979; Thurlow \& Erchul, 1977). Other experiments have shown, however, that experimental conditions, musical context, and other factors also

This research was supported by NINCDS Grant NS1540502. I thank George Boggs, Edward Carney, Lawrence Feth, and William Hartmann for comments on a previous version of this manuscript. can have a large effect on the degree to which octave equivalence is manifest. For example, there are no apparent octave equivalence effects for open-set identification of melodies when notes are randomly assigned to different octaves (Deutsch, 1972, 1978), whereas, for closed-set identification of melodies whose notes are randomly assigned to different octaves, with the restriction that melodic contour be maintained, octave equivalence effects apparently play a large role (Idson \& Massaro, 1978).

One study often cited as providing convincing evidence for octave equivalence that is independent of musical training was reported by Shepard (1964). He synthesized complex tones composed of partials separated by octaves that were amplitude weighted such that the tones were, in essence, passed through a constant-center-frequency cosine-shaped bandpass filter. These tones have a property such that a change in fundamental frequency of an octave results in an exact replication of the spectral pattern (with the exception of the lowest and highest partials, which are, however, highly attenuated because of the amplitude weighting). Relative pitch judgments between pairs of these complex tones that differed in fundamental frequency showed that tones with fundamental frequencies less than (roughly) one-half octave above the reference tone were judged higher in pitch, whereas tones between one-half and one octave above were judged lower in pitch. These results were explained by Shepard (1964) in terms of tracking of individual partials (analytic listening) and the proximity principle - that is, relative pitch judgments tend to be made with respect to the nearest partial. Since for fundamental frequency increases of more than half an octave the nearest partial is lower in frequency, this will be heard as a decrease in pitch. Terhardt (1979) has recently pointed out that a similar result would be expected from synthetic listening- 
that is, hearing a unitary low pitch corresponding to a (pseudo)fundamental frequency derived from the resolved partials of the complex tone. Due to the influence of a dominant region for resolved partials that will elicit such a pitch, the nominal fundamental pitch for the complex tones used by Shepard is much higher than the fundamental frequency and shows a sawtooth pattern, increasing with increasing fundamental frequency but with sudden octave jumps down. Thus, Shephard's results for pitch judgments between pairs of these complex tones are not particularly surprising in terms of either mode of listening. These experiments have recently been expanded upon by Pollack (1978).

The aspect of Shepard's paper that is surprisingand the aspect that is cited as evidence for octave equivalence-is the auditory illusion that obtains when a sequence of these complex tones is played in which fundamental frequency is incremented over the range of an octave (e.g., in 1-semit steps) and the whole cycle is played repeatedly. The impression is that of a sequence of pitch steps that are (for increasing fundamental frequency) always upward; however, subjects report that, contrary to expectation, the overall pitch "never gets higher." Shepard likened the effect to the visual "stairway to heaven" illusion. The reasoning behind citing the illusion as evidence for octave generalization, although never elucidated, is presumably as follows: If it is assumed that the observer tracks a single partial (analytic listening), then, given the results of the relative pitch judgments, at the end of each cycle he would be expected to switch to the next highest partial (following the proximity principle). This listening strategy would lead to a sequence of continually rising pitch steps. However, given the bandpass filtering, a point will be reached at which this partial will no longer be audible, leading to a jump to a partial that lies one or more octaves below. If it is assumed, on the other hand, that the observer listens synthetically, the observed fundamental pitch would, as mentioned previously, still be expected to follow the same kind of sawtooth pattern: continuously increasing in small steps but with sudden downward octave jumps. Since these downward jumps in pitch of an octave (corresponding to either listening mode) are not heard, and the pitch appears to always rise, it is concluded that this is a result of octave equivalence-that is, the similarity between tones separated by an octave results in the downward octave pitch jumps not being noticed. Since the illusion seems to be independent of musical training, it is taken to be stronger evidence of octave equivalence than the previously cited paperswhich seem to imply that octave equivalence is learned as a consequence of musical training.

In this paper, we will demonstrate that this illusion obtains for a more general set of complex tones- that is, tones in which the partials are separated by equal ratios (including, but not limited to, octaves) and for which spectral structure is cyclically repeated as a function of fundamental frequency. Hence, the illusion is not attributable to octave equivalence.

\section{METHOD}

\section{Stimuli and Apparatus}

The complex tones were computer synthesized at a sampling rate of $10 \mathrm{kHz}$. The frequencies of the partials of a single tone are given by

$$
f_{n}=f \cdot n^{s},
$$

where $\mathrm{f}$ is the fundamental frequency of the tone, $\mathrm{n}$ is the partial number $n=1,2,4,8, \ldots$, and $s$ is the "stretch exponent" (see Slaymaker, 1970). This equation yields complex tones whose partials are separated by equal ratios; for $s=1$, the tones are equivalent to those used by Shepard (1964)-that is, the partials are separated by octaves. For other values of $s$, the partials are separated by ratios that are "stretched" (s $>1)$ or "compressed" $(s<1)$ relative to an octave, in which case the tones are, in general, inharmonic. A property of these tones is that, as in the Shepard demonstration, the spectral pattern of the partials is unchanged (with the exception, of course, of the lowest and highest partials) for changes in fundamental frequency equal to the partial separation (see Figure 1). Therefore, a cyclical sequence of tones can be constructed by varying fundamental frequency over a range equal to the partial separation. The cyclical pattern in Shepard's demonstration consisted of the equitempered chromatic scale-that is, the octave partial separation was traversed in 12 equal semit (100 cent) steps. In our study, fundamental frequency is also varied in 100-cent steps, but, since partial separation is in most cases greater or less than an octave, there are proportionally more or less than 12 tones in a cycle for these cases.

The parameters for the stimulus conditions used in this experiment are listed in Table 1. The stimulus condition OCT refers to

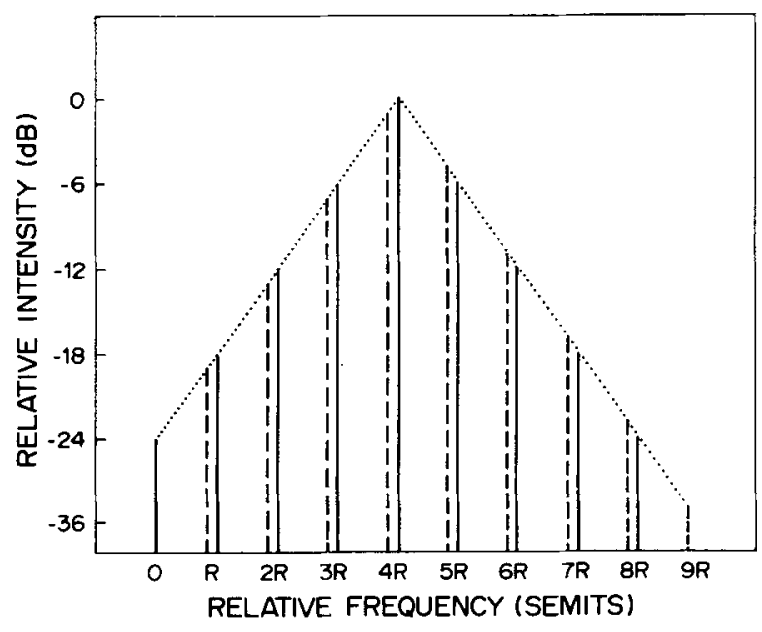

Figure 1. A graphic representation of the spectrum of the tones used to generate the illusion. The solid lines represent the partials of a tone with a fundamental frequency of $15 \mathrm{~Hz}$ and an arbitrary partial separation of $\mathbf{R}$ semits. The dashed lines represent the partials of a tone with the same partial separation but with a fundamental frequency slightly less than $R$ semits above $15 \mathrm{~Hz}$. The dotted lines represent the envelope of the spectrum. 
Table 1

Parameters for Stimulus Conditions

\begin{tabular}{lrrrr}
\hline $\begin{array}{l}\text { Stimulus } \\
\text { Condition }\end{array}$ & $\mathbf{R}$ & \multicolumn{1}{c}{$\mathbf{P}$} & $\mathbf{S}$ & $\mathbf{T}$ \\
\hline C2S & 10 & 10 & 1 & 10 \\
OCT & 12 & $9-8$ & 1 & 12 \\
OCT(2S) & 12 & $9-8$ & 2 & 6 \\
S2S & 14 & $8-7$ & 1 & 14 \\
C4S & 8 & $13-12$ & 1 & 8 \\
S4S & 16 & $7-6$ & 1 & 16 \\
C6S & 6 & $17-16$ & 1 & 6 \\
\hline
\end{tabular}

Note $-R=$ partial separation (in semits); $P=$ number of partials; $S=$ ratio between consecutive tones (in semits); $T=$ number of tones in cycle.

octave (12 semits) partial separation, $\mathrm{C} 2 \mathrm{~S}$ refers to compression by two semits (partial separation of 10 semits), S2S refers to stretching by two semits (separation of 14 semits), and so on. The $10-\mathrm{kHz}$ sampling rate imposed an upper frequency bound of $5 \mathrm{kHz}$. Because of this limitation, it was necessary to omit the highest partial if it rose above $5 \mathrm{kHz}$ as fundamental frequency was increased. This explains the two numbers in the column labeled "number of partials" for some of the stimulus conditions. The amplitude of the partials was weighted by a simple triangular weighting function: $-6 \mathrm{~dB}$ per octave relative to $240 \mathrm{~Hz}$. Pollack (1978) showed that this type of weighting function was adequate to replicate Shepard's (1964) findings for relative pitch judgments. The fundamental frequency for the lowest tone in the sequence was $15 \mathrm{~Hz}$ for all stimulus conditions. A graphic illustration of the complex tone (for an arbitrary separation of partials of $\mathbf{R}$ semits) is given in Figure 1. The tones were $100 \mathrm{msec}$ in duration, including a 10-msec (half-cosine) rise and decay. They were played via a 12-bit D/A through TDH-39 headphones in a soundattenuated room. The stimuli were presented at an intensity such that the level of a partial at $240 \mathrm{~Hz}$ (i.e., 0 weighting attenuation) was $65 \mathrm{~dB}$ SPL.

\section{Subjects}

The subjects were recruited from the Purdue student body and were paid \$3.00 for participating. Although musical training was not a criterion for recruitment, each subject filled out a short questionnaire on his/her musical background and training. Twentyone subjects completed the entire set of stimulus conditions.

\section{Procedure}

In the introduction, it was noted that the expected percept for the cyclically ascending tone sequences described above would be a stepwise increase in pitch over the cycle with sudden octave decreases in pitch between cycles. This is, in fact, what Shepard's (1964) subjects reported when there was no silent interval between consecutive tones; in order for the illusion of continually ascending pitch to obtain, it was necessary to leave a relatively long (several hundred milliseconds) silent interval between tones. ${ }^{1}$ This downward jump in pitch was used as a criterion for judging whether or not the subjects heard the illusion. The subjects were presented with 10 cycles of each of the seven stimulus conditions listed in Table 1 with an intertone interval of $800 \mathrm{msec}$. The seven stimulus conditions were presented in random order. Subjects were told that they would hear a sequence of tones that would at first increase in pitch continuously, but that at certain points in the sequence there might be sudden large decreases in pitch, after which the sequence would again increase in pitch. The subjects were instructed to press a button on a response panel whenever they heard a decrease in pitch. The number of the cycle and the number of the tone within the cycle at which these responses occurred were recorded by the computer. After completing the seven stimulus conditions with the 800 -msec intertone interval, the subjects were presented with three of the stimulus conditions (C2S, OCT, and S2S) with an intertone interval of $50 \mathrm{msec}$, after which they again heard the three stimulus conditions with an 800 -msec delay between tones.

\section{RESULTS AND DISCUSSION}

The results were first analyzed by plotting histograms (number of responses at each tone in the cycle) for each subject for each stimulus condition. A perusal of these histograms showed that there were large differences in response patterns among the subjects. This variation in response patterns for a given stimulus condition could be roughly categorized into three response modes: (1) no (or very few) responses over all 10 cycles of a stimulus presentation, a strong indication that these observers experienced the illusion of continually rising pitch; (2) one response per cycle at the same tone in the cycle, a clear indication that these subjects detected the cyclical nature of the stimuli; and (3) a relatively large number of responses (about one per cycle) randomly distributed over all of the tones in the cycle, an ambiguous condition in which the subjects might have detected downward pitch jumps but at different tones in each cycle or may simply have been responding at random.

A synopsis of the results is presented in Table 2. The first column (NR) under each stimulus condition gives the total number of responses (over the 10-cycle presentation length) for each subject, while the second column (MR) under each stimulus condition gives the total number of responses at the modal value of the response histogram (if this value was 4) and, in parentheses $(T)$, the tone in the cycle at which this modal value occurred. Thus, a small value in column 1 across conditions would be categorized as response mode 1 (e.g., Subjects 1, 15, 18, and 19). A large number of responses in column 1 that are seen in column 2 to occur predominantly at one tone in the cycle are indicative of response mode 2 (e.g., Subjects 7, 12, and 21). Finally, a large number of responses in column 1 along with no entry in column 2 would imply response mode 3 .

The primary conclusion to be drawn from the results in Table 2 is that there is no essential difference between the response to conditions in which the partials are separated by octaves and those in which the partials are separated by other ratios. Although there are differences across conditions, subjects tend to stay in a single response mode-that is, subjects who hear the illusion in one condition tend to hear it for all conditions, whereas subjects who detect the cyclical pattern also tend to do so for all conditions. In particular, there were no subjects who had a small number of responses in column 1 (indicating that they heard the illusion) for one of the octave conditions who did not also have a small number of re- 
Table 2

Synopsis of Results of Response Histograms for Individual Subjects

\begin{tabular}{|c|c|c|c|c|c|c|c|c|c|c|c|c|c|c|c|c|c|c|c|c|c|c|c|c|c|c|c|c|c|c|}
\hline \multirow{3}{*}{$\begin{array}{l}\text { Sub- } \\
\text { ject }\end{array}$} & \multicolumn{30}{|c|}{ Stimulus Condition } \\
\hline & \multicolumn{3}{|c|}{$\mathrm{C} 6 \mathrm{~S}$} & \multicolumn{3}{|c|}{$\mathrm{C} 4 \mathrm{~S}$} & \multicolumn{3}{|c|}{$\mathrm{C} 2 \mathrm{~S}$} & \multicolumn{3}{|c|}{ OCT } & \multicolumn{3}{|c|}{$\mathrm{OCT}(2 \mathrm{~S})$} & \multicolumn{3}{|c|}{$\mathbf{S} 2 \mathrm{~S}$} & \multicolumn{3}{|c|}{ S4S } & \multicolumn{3}{|c|}{$\mathrm{C} 2 \mathrm{~S}(\mathrm{II})$} & \multicolumn{3}{|c|}{ OCT(II) } & \multicolumn{3}{|c|}{ S2S(II) } \\
\hline & NR & MR & $\mathrm{T}$ & NR & MR & & NR & MR & & NR & MR & & NR & MR & & NR & & $T$ & NR & MR & $\mathbf{T}$ & NR M & & $\mathrm{T}$ & NR & MR & $\mathbf{T}$ & NR & MR & $\mathrm{T}$ \\
\hline 1 & 0 & & & 0 & & & 0 & & & 0 & & & 3 & & & 0 & & & $\mathbf{0}$ & & & 1 & & & 2 & & & 0 & & \\
\hline 2 & 3 & & & 9 & 7 & 2 & 9 & & & 8 & 5 & 9 & 10 & 7 & 5 & 10 & 4 & 2 & 13 & & & 8 & 5 & 1 & 9 & 9 & 9 & 9 & & \\
\hline 3 & 9 & & & 8 & 4 & 1 & 0 & & & 11 & 5 & 9 & 8 & & & 14 & 4 & 7 & 6 & & & 13 & 5 & 1 & 12 & 5 & 6 & 516 & 5 & \\
\hline 4 & 9 & & & 13 & & & 11 & & & 14 & 5 & 9 & 10 & 6 & 5 & 11 & & & 22 & 5 & 9 & 14 & 4 & 9 & 17 & 4 & 5 & 19 & 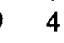 & 10 \\
\hline 5 & 8 & 5 & 5 & 7 & 4 & 6 & 7 & & & 12 & 6 & 9 & 6 & 4 & 5 & 5 & & & 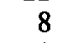 & 5 & 11 & 8 & & & 10 & 7 & 6 & 58 & 3 & 20 \\
\hline 6 & 4 & & & 1 & & & 1 & & & 7 & & & 7 & 4 & 6 & 6 & & & 8 & 4 & 14 & 8 & & & 13 & 4 & 7 & 12 & ? & \\
\hline 7 & 8 & 8 & 6 & 9 & 9 & 6 & 8 & 7 & 1 & 8 & 8 & 6 & 7 & 7 & 4 & 9 & 9 & 4 & 10 & 10 & 6 & 9 & 9 & 1 & 9 & 9 & 6 & 5 & 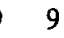 & \\
\hline 8 & 9 & 6 & 6 & 10 & 9 & 6 & 0 & & & 4 & & & 10 & 9 & 5 & 9 & 8 & 4 & 6 & 6 & 6 & 6 & & & 9 & 9 & 6 & 5 & 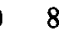 & \\
\hline 9 & 9 & & & 6 & 4 & 6 & 0 & & & 9 & 6 & 6 & 11 & 8 & 4 & 4 & & & 4 & & & 10 & 8 & 1 & 13 & 7 & 6 & 5 & . & \\
\hline 10 & 8 & & & 5 & & & 0 & & & 1 & & & 9 & 7 & 2 & 7 & & & 7 & & & 12 & 5 & 4 & 15 & 4 & 2 & 2 & 3 & \\
\hline 1 & 8 & 4 & 3 & 11 & 4 & 7 & 9 & & & 8 & & & 7 & & & 14 & & & 15 & & & 13 & 4 & 10 & 12 & & & 5 & 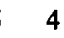 & \\
\hline 12 & 9 & 7 & 6 & 8 & 7 & 6 & 7 & 6 & 1 & 10 & 9 & 6 & 11 & 9 & 4 & 6 & 6 & 4 & 8 & 8 & 6 & 9 & 9 & 1 & 9 & 5 & 6 & 10 & 9 & \\
\hline 13 & 9 & 4 & 5 & 11 & & & 13 & & & 16 & & & 7 & 4 & 1 & 3 & & & 16 & & & 12 & & & 12 & & & 17 & 6 & 13 \\
\hline 14 & 12 & 5 & 6 & 9 & & & 9 & 4 & 1 & 10 & 5 & 9 & 8 & 4 & 4 & 6 & 4 & 8 & 8 & 4 & 2 & 6 & & & 10 & 5 & 9 & 12 & 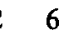 & 8 \\
\hline 15 & 0 & & & 0 & & & 0 & & & 0 & & & 2 & & & 0 & & & 2 & & & 9 & 4 & 3 & 10 & 10 & 6 & 9 & 8 & \\
\hline 16 & 0 & & & 4 & & & 2 & & & 1 & & & 4 & & & 2 & & & 6 & & & 5 & & & 6 & & & 9 & 4 & \\
\hline 17 & 0 & & & 0 & & & 6 & & & 3 & & & 9 & 4 & 5 & 0 & & & 13 & 6 & 11 & 6 & & & 9 & 5 & 6 & 10 & 5 & 14 \\
\hline 18 & 0 & & & 0 & & & 0 & & & 0 & & & 0 & & & 0 & & & 0 & & & 0 & & & 0 & & & 0 & & \\
\hline 19 & 0 & & & 0 & & & 0 & & & 0 & & & 0 & & & 0 & & & 0 & & & 1 & & & 0 & & & 5 & & \\
\hline 2 & 3 & & & 6 & & & 5 & & & 2 & & & 6 & 4 & 2 & 5 & & & 9 & 4 & 4 & 7 & 4 & 9 & 12 & 4 & 10 & 6 & & \\
\hline 21 & 11 & 9 & 6 & 8 & 6 & 6 & 9 & 9 & 1 & 9 & 6 & 6 & 10 & 7 & 4 & 9 & 7 & 4 & 15 & 8 & 6 & 9 & 9 & 1 & 9 & 9 & 6 & 10 & 10 & 4 \\
\hline $0 \mathrm{NR}$ & 6 & & & 5 & & & 8 & & & 4 & & & 2 & & & 5 & & & 3 & & & 1 & & & 2 & & & 2 & & \\
\hline$<5$ NR & 9 & & & 7 & & & 10 & & & 9 & & & 5 & & & 8 & & & 5 & & & 3 & & & 3 & & & 2 & & \\
\hline$<4 \mathrm{MR}$ & & 13 & & & 12 & & & 17 & & & 12 & & & 7 & & & 14 & & & 11 & & & 10 & & & 6 & & & 5 & \\
\hline
\end{tabular}

Note $-N R=$ total number of responses over 10 presentation cycles; $M R=$ number of responses at the modal value of the response histogram; $T=$ tone in cycle at which modal value occurred.

sponses in column 1 for some of the other conditions. Risset (Note 2) has also produced the illusion with tones having nonoctave partial separation. These results are a clear demonstration that octave equivalence is not the basis for the illusion.

Only about a third of the subjects fall into response mode 1 , in which it is fairly certain they hear the illusion. This is probably because the procedure biases them against hearing the illusion (i.e., they are cued to listen for something other than a continuous sequence of upward pitch steps). In informal listening sessions, 20 other observers were played samples of the same stimuli and were simply asked to report what they heard (as in Shepard's original demonstration). All but one reported hearing a continually rising pitch. Another factor that may have contributed to many subjects' detecting the cyclical nature of the stimuli was the fact that it was necessary to omit partials higher than $5 \mathrm{kHz}$. At the levels at which the tones were presented, an isolated $5-\mathrm{kHz}$ tone would have been above threshold for an observer with normal hearing sensitivity. The results of the observers who fell into response mode 2 show that, in most cases, the tone in the cycle at which the modal value of the responses occurred was the tone at which the highest partial was first omitted as fundamental fre- quency was increased. This is consistent with the subjective impressions of a number of these observers who said that they heard a continually rising pitchbut detected a cyclic change in quality.

There was no obvious correlation between the response mode of a given subject and his/her degree of musical training. There was also no indication that the musically trained subjects found the illusion more salient for the octave-partial-separation condition vis-à-vis the nonoctave conditions. Given the high correlation between musical training and degree of octave similarity shown in the other studies, the lack of correlation in this study further suggests that octave similarity is not a factor in the illusion.

In addition to the large intersubject differences in responses, smaller, but distinct, differences exist across conditions. These differences may provide a hint as to the parameters that are important for producing the illusion. The bottom three rows in Table 2 show the total number of subjects for each condition who showed zero responses, fewer than five total responses, and fewer than four total responses on any given tone. The assumption is that the larger these values are, the more often the illusion was heard. Considering the seven conditions that were run initially, there appears to be a tendency for an increased 
saliency of the illusion for the compressed-partials conditions; $\mathrm{C} 2 \mathrm{~S}$ is clearly the condition for which the illusion is most salient. However, the saliency of the illusion apparently decreases with a smaller number of tones in the cycle, as indicated by the difference between OCT and OCT (2S); in the latter condition, the partials are separated by octaves but the cycle contains 6 two-semit steps rather than 12 semit steps. Since the conditions with the more compressed partials also have fewer tones in the cycle, there is a confounding of these two effects.

For the compressed-partial condition, a greater tendency would be expected for synthetic-mode listening by the subjects. Although some degree of resolution of partials is necessary for synthetic pitch perception (Houtsma \& Goldstein, 1972), wide separation of partials favors analytic pitch perception (Houtsma, 1979). Thus, the tendency for greater saliency of the illusion for tones with compressed partials may imply that it is related more to the synthetic than to the analytic mode. However, the key factor for the illusion may simply be the ambiguity of the pitch in either mode, combined with the nearly cyclic nature of the stimuli. For both the analytic mode and the synthetic mode there is no single pitch associated with the stimulus, but many possible pitches. In the case of the analytic mode, the progressively wider spacing (in hertz) of the higher partials will result in the upper four or five partials' being resolvable; in the case of the synthetic mode, there will be a number of "estimates" of the fundamental pitch that might be heard (e.g., see Houtsma, 1979). The task is then essentially a multichannel listening task, rather than the tracking of a single pitch, with a majority of the channels (following the proximity principle) showing an increase in pitch for each successive tone-including the increment from the last to the first tone in the cycle.

There is also a clear learning effect in the perception of the illusion. Conditions C2S(II), OCT(II), and S2S(II) denote repetitions of Conditions C2S, OCT, and S2S after the subjects had heard the same conditions with an intertone interval of $50 \mathrm{msec}$. For the 50-msec conditions, most of the observers reported hearing the cyclic pattern, and this was evident in the histograms of the responses (although the speed of presentation combined with the response times of the subjects resulted in a smearing of the modal value across several tones). The results for C2S(II), OCT(II), and S2S(II) in Table 2 show that, presumably as a result of their experience of the cyclical nature of the stimuli in the 50-msec condition, the illusion was heard much less often than in the initial presentation of C2S, OCT, and S2S. This learning effect was, in fact, noted in the presentation of the initial seven stimulus conditions; several subjects picked up the cyclical pattern after two or three conditions had been presented.
The conclusion that the Shepard illusion is not evidence for octave equivalence does not imply that the concept of chroma is invalid. It seems fairly clear that the bidimensional models are an appropriate description of the perception of relative pitch under certain circumstances and/or by persons with extensive musical training. The main question is whether octave equivalence is entirely a result of learning and experience or if there is some basis for it inherent in the operation of the auditory system. One experiment that is often cited as evidence for octave equivalence and that is clearly independent of musical training is the octave generalization in rats reported by Blackwell and Schlosberg (1943). There are, however, a number of problems with this experiment, for example, harmonic distortion in the stimulus, and it has apparently never been replicated.

There is, on the other hand, a good deal of physiological and psychophysical evidence that would, under certain circumstances, imply a uniqueness for octave relationships. For example, if pure tone pitch is, as some evidence indicates, primarily coded in the synchrony of neural discharges to the stimulus frequency (e.g., by interspike intervals), then there is an obvious physiological relationship between tones whose frequencies are related by small-integer ratios. Also, the process of complex-tone pitch perception, which can be modeled as the best fit of a harmonic series to the partials of the stimulus (e.g., Gerson \& Goldstein, 1978), leads to octave ambiguities in fundamental pitch estimates for many situations. Finally, sensory dissonance, which is related to roughness and beats (see Terhardt, 1978), is not increased by the simultaneous addition of a harmonic complex tone an octave higher in fundamental frequency. These latter two effects, however, may have had more to do with the original formulation of the musical scales of a given culture than they have with playing a predominant role in the everyday perception of pitch relationships. The preponderance of evidence indicates (e.g., see Burns \& Ward, in press) that musical interval judgments, including the octave, are based on the learned categories of the individual's musical culture.

\section{REFERENCE NOTES}

1. Risset, J. Auditory demonstration tapes, Laboratory of Psychophysics, Harvard University, December 15, 1978.

2. Risset, J. Personal communication, 1980.

\section{REFERENCES}

Allen, D. Octave discriminability of musical and non-musical subjects. Psychonomic Science, 1967, 7, 421-422.

Blackwell, H. R., \& Schlosberg, H. Octave generalization, pitch discrimination, and loudness thresholds in the white rat. Journal of Experimental Psychology, 1943, 33, 407-419.

Burns, E. M., \& WARD, W. D. Intervals, scales and tuning. In 
D. Deutsch (Ed.), The psychology of music. New York: Academic Press, in press.

Deutsch, D. Music recognition. Psychological Review, 1969, 76, 300-307.

Deutsch, D. Octave generalization and tune recognition. Perception \& Psychophysics, 1972, 11, 411-412.

DeUTBCH, D. Octave generalization and melody identification. Perception \& Psychophysics, 1978, 23, 91-92.

Gerson, A., \& Goldste in, J. L. Evidence for a general template in central optimal processing for pitch of complex tones. Journal of the Acoustical Society of America, 1978, 63, 498-510.

Houtsma, A. J. M. Musical pitch of two-tone complexes and predictions by modern pitch theories. Journal of the Acoustical Society of America, 1979, 66, 87-99.

Houtsma, A. J. M., \& Goldstein, J. L. The central origin of the pitch of complex tones: Evidence from musical interval recognition. Journal of the Acoustical Society of America, 1972, $51,520-529$.

IDson, W. L., \& MAssaro, D. A bidimensional model of pitch in the recognition of melodies. Perception \& Psychophysics, $1978,24,551-565$.

Krumhan8l, C. L., \& Shepard, R. N. Quantification of the hierarchy of tonal functions within a diatonic context. Journal of Experimental Psychology: Human Perception and Performance, 1979, 5, 579-594.

Pollack, I. Decoupling of auditory pitch and stimulus frequency: The Shepard demonstration revisited. Journal of the Acoustical Society of America, 1978, 63, 202-206.
Revesz, G. An introduction to the psychology of music. Norman: Oklahoma University Press, 1954.

SHEPARD, R. N. Circularity of judgments of relative pitch. Journal of the Acoustical Society of America, 1964, 36, 2346-2353.

Slaymake R, F. H. Chords from tones having stretched partials. Journal of the Acoustical Society of America, 1970, 47, 1569. 1571.

Stevens, S. S., \& Volkmann, J. The relation of pitch to frequency: Revised scale. American Journal of Psychology, 1940, 53, 329-353.

Terhardt, E. Psychoacoustic evaluation of musical sounds. Perception \& Psychophysics, 1978, 23, 483-492.

Terhardt, E. Calculating virtual pitch. Hearing Research, 1979, 1, 155-182.

Thurlow, W. R., \& Erchul, W. P. Judged similarity in pitch of octave multiples. Perception \& Psychophysics, 1977, 22, 177-182.

\section{NOTE}

1. It should be noted, however, that Risset (Note 1) has produced the illusion for the case of a continually changing fundamental frequency that is, in one sense, the limiting case of a short interval between consecutive tones in the cycle.

(Manuscript received May 1, 1981; revision accepted for publication July $27,1981$. 